\section{CANCER INCIDENCE AMONG NORDIC FIREFIGHTERS}

Paul Demers, ${ }^{1}$ Jan Ivar Martinsen, ${ }^{2}$ Elisabete Weiderpass, ${ }^{3}$ Kristina Kjærheim, ${ }^{2}$ Elsebeth Lynge, ${ }^{4}$ Pär Sparén, ${ }^{3}$ Eero Pukkala ${ }^{5}$ Occupational Cancer Research Centre, Toronto, Canada; ${ }^{2}$ Cancer Registry of Norway, Oslo, Norway; ${ }^{3}$ Karolinska Institute, Stockholm, Sweden; ${ }^{4}$ University of Copenhagen, Copenhagen, Denmark; ${ }^{5}$ Finnish Cancer Registry, Helsinki, Finland

10.1136/oemed-2011-100382.60

Objectives Firefighters are potentially exposed to known and suspected carcinogens, including fire combustion products, vehicle emissions, and shift work. However, their risk of cancer remains controversial. The objective of this study was to examine their risk of cancer among firefighters using data from the Nordic Occupational Cancer (NOCCA) study.

Methods The study population consisted of 16223 men, age 30-64, who reported being firefighters in the 1960, 1970, 1980 and/or 1990 censuses in Denmark, Finland, Norway and Sweden. These data were linked with national tumour registry data. Standardised incidence ratios (SIRs) and 95\% CIs were calculated with expected numbers based on country, sex, age, and period.

Results Altogether 2470 cancer cases were observed in the follow-up up to 2005. Excesses of non-melanoma skin cancer ( $\mathrm{SIR}=1.33, \mathrm{CI}=1.11$ to 1.59 ), lung adenocarcinoma (SIR=1.31, $\mathrm{CI}=1.04$ to 1.63 ), malignant melanoma ( $\mathrm{SIR}=1.25, \mathrm{CI}=1.03$ to 1.51$)$, colon cancer ( $\mathrm{SIR}=1.15, \mathrm{CI}=1.00$ to 1.32 ), prostate cancer ( $\mathrm{SIR}=1.14, \mathrm{CI}=1.05$ to 1.23 ), and mesothelioma ( $\mathrm{SIR}=1.56, \mathrm{CI}=0.91$ to 2.50$)$ were observed. Risks of mesothelioma ( $\mathrm{SIR}=2.61, \mathrm{CI}=1.25$ to 4.80 ), lung adenocarcinoma ( $\mathrm{SIR}=1.92, \mathrm{CI}=1.35$ to 2.65$)$, and non-melanoma skin cancer $(\mathrm{SIR}=1.41, \mathrm{CI}=1.11$ to 1.77$)$ were highest among firefighters over 70. SIRs for prostate cancer (SIR=2.61, CI=1.35 to 4.56) and melanoma (SIR=1.63, CI=1.15 to 2.24) were highest among those under age 50 . 


\section{Abstracts}

Conclusions The small overall excesses for most sites are consistent with recent meta-analyses, with the exception of mesothelioma and lung adenocarcinoma, which may not have been previously observed because of a lack of power and histologic data in previous studies. 